\title{
La construction du risque urbain en périphérie nord-est de Cuzco (Pérou)
}

La construcción del riesgo urbano en la periferia noreste de Cuzco (Perú)

The construction of urban risk in the north-east perifery of Cuzco (Peru)

\section{Nicolas Rey}

\section{QpenEdition}

\section{Journals}

Édition électronique

URL : http://journals.openedition.org/bifea/3870

DOI : 10.4000/bifea.3870

ISSN : 2076-5827

Éditeur

Institut Français d'Études Andines

Édition imprimée

Date de publication : 1 août 2007

Pagination : 259-276

ISSN : 0303-7495

Référence électronique

Nicolas Rey, "La construction du risque urbain en périphérie nord-est de Cuzco (Pérou) », Bulletin de I'Institut français d'études andines [En ligne], 36 (2) | 2007, mis en ligne le 01 février 2008, consulté le 03 décembre 2020. URL : http://journals.openedition.org/bifea/3870 ; DOI : https://doi.org/10.4000/ bifea.3870

\section{(c) (i) $\odot$}

Les contenus du Bulletin de l'Institut français d'études andines sont mis à disposition selon les termes de la licence Creative Commons Attribution - Pas d'Utilisation Commerciale - Pas de Modification 4.0 International. 


\title{
La construction du risque urbain en périphérie nord-est de Cuzco (Pérou)
}

\author{
Nicolas Rey*
}

\begin{abstract}
Résumé
Dans l'ancienne capitale inca, Cuzco, les pentes abruptes ont été colonisées par les habitants pauvres n'ayant pas trouvé à se loger dans le centre-ville. Située dans une zone de forte activité sismique, Cusco a ainsi vu ces dernières décennies deux types de catastrophes se succéder : le tremblement de terre, comme celui de 1950, a déplacé la population vers les périphéries, qui se sont ensuite étendues sans planification; et depuis les années 1980, les glissements de terrain emportent les maisons en adobe occupées en majorité par des populations indiennes et métis vulnérables. Face à l'urbanisation des hommes, la Terre Mère, la Pacha Mama, ne serait-elle pas de retour, pour se venger ? Cet article montre dans un premier temps comment une expansion urbaine non maîtrisée par les autorités a conduit à la construction du risque en périphérie de Cuzco. On rentre ensuite dans le détail des stratégies développées par les habitants quechuas et métis pour lesquels la recherche du moindre coût en se logeant le long des pentes abruptes se fait au prix d'un risque plus grand en partie assumé. Enfin, entre positions contradictoires des autorités selon les niveaux décisionnels (Defensa civil, mairie...), et représentation du risque par les habitants renvoyant aux schèmes de la culture quechua, il s'agit alors de confronter les différentes stratégies des acteurs face au risque pour espérer en améliorer sa gestion.
\end{abstract}

Mots clés : risques, quartiers périphériques, gouvernance, anthropologie, Cuzco

\section{La construcción del riesgo urbano en la periferia noreste de Cuzco (Perú)}

\section{Resumen}

En la antigua capital Inca, Cuzco, las laderas fueron colonizadas por los habitantes pobres que no encontraron donde vivir en el centro de la ciudad. Situada en una zona de fuerte actividad sísmica, Cusco ha conocido dos peligros en las ultimas décadas: el terremoto de 1950 que provocó el traslado

* Docteur en sociologie du développement (La Sorbonne \& IEDES) : 18, rue de la Tour, 77410 Villevaudé. E-mail: nicolartiste@yahoo.com 
de la población hacia las periferias que se han desarrollado sin planificación y los derrumbes que desde los años 1980 han destruido muchas casas de adobe ocupadas por indígenas o mestizos vulnerables. Uno se pregunta si frente al proceso de urbanización que llevan a cabo los hombres, la Madre Tierra, la Pacha Macha, no ha vuelto para vengarse. Este artículo demuestra cómo la expansión urbana no planificada por las autoridades ha desembocado en la construcción en condiciones de riesgo en las periferias de Cuzco. Luego se trata de entender en detalle las estrategias económicas desarrolladas por los habitantes quechuas y mestizos que buscan alquileres más accesibles, pero que aceptan instalarse en zonas altamente peligrosas como son las laderas, por falta de recursos. En fin, se trata de confrontar las estrategias de los diferentes actores para mejorar la gestión del riesgo, frente a las contradicciones de las diversas autoridades (Defensa civil, municipalidad...) y la representación del riesgo que tienen los habitantes quechuas.

Palabras clave: riesgos, suburbios, gobernabilidad, antropología, Cuzco

\title{
The construction of urban risk in the north-east perifery of Cuzco (Peru)
}

\begin{abstract}
In the ancient capital of the Inca Empire, Cuzco, the steep slopes have been occupied by poor inhabitants who didn't find anywhere to live in the city center. Localized in a zone of great seismic activity, Cuzco has known two types of disasters in the last decades: earthquakes, like the one of 1950, that deplaced the population to the peripheries without planning; and landslides, which since the 1980's, destroyed the adobe houses occupied by a majority of indian and mestizo population. Wouldn't the Earth Mother, The Pacha Mama, come back, to exact revenge, after seeing the results of men's urbanisation? In the first part, this article explains how the uncontrolled urban expansion has led to the construction of risk in the peripheries of Cuzco ; then, in the second part, we detail strategies developed by the quechua-speaking inhabitants and mestizos who are looking for cheaper housing prices on the high risk slopes ; finally, we examine examine contradictory positions of the authorities at diferent levels ( civil defense, municipality...), and the representation of risk made by the inhabitants within the context the quechua culture. We conclude by considering the strategies of the actors to mitigate the risk and improve its management.
\end{abstract}

Keywords: risks, suburbs, governance, anthropology, Cuzco

Dans les villes de montagne latino-américaines, et en particulier andines, les glissements de terrain se succèdent depuis plusieurs décennies dans les quartiers périphériques en pente. Ces catastrophes surviennent dans le contexte socio-historique et politique suivant : crise du monde rural et explosion urbaine après 1950, absence d'une planification capable d'organiser l'expansion de la ville, classes populaires organisées en associations pour se loger, dans les espaces restés libres, en général les plus ingrats, et régularisation après-coup par les autorités de ces zones à risque, une fois que le «mal » a été fait.

La ville de Cuzco, située dans les Andes péruviennes, à 3500 mètres d'altitude, n'échappe pas à ces constantes. 
Jusque dans les années 1950, Cuzco, ancienne « capitale » inca, était principalement délimitée par son tracé colonial. Cuzco était la ville la plus dense du Pérou: le phénomène dit de tugurizacion dominait (surconcentration et dégradation des quartiers ainsi occupés). II n'existait alors pas de continuité urbaine entre le centre historique proprement dit, et les autres districts plus éloignés ${ }^{1}$. Suite au tremblement de terre de 1950, qui ravagea plus de 3000 maisons, laissant sans toit de 30000 à 40000 personnes, Cuzco va s'étendre vers l'est. Mais ce début de continuité urbaine se fera au prix d'une installation le long des laderas (pentes abruptes) dominant la ville, sans planification urbaine. La municipalité de Cuzco va régulariser après coup ces nombreux quartiers construits sur des terrains rachetés par les habitants aux ordres religieux et aux grands propriétaires (hacendados).

Ainsi, en plus du centre historique, toujours menacé par l'activité sismique intense dans la région, sont apparues le long des pentes de nouvelles zones à risque, nées de l'installation non contrôlée d'habitants qui ont élaboré leur système de drainage et une trame urbaine sans cohérence avec la ville plus formelle. Résultat : de nombreux glissements de terrains affectent régulièrement depuis vingt ans la périphérie nord-est de Cuzco, emportant maisons et ruelles, dans la chute. Deux glissements de terrain survenus respectivement en avril 2001 et janvier 2002 dans le secteur dit « comité 6 » du quartier Asociacion Pro-Vivienda Los Incas2, provoquèrent la destruction de cinq maisons, et endommagèrent deux autres constructions limitrophes. La rue centrale du comité 6, constituée d'un escalier, a également été emportée, pour moitié. En réponse, les autorités s'accordèrent pour déclarer qu'il fallait évacuer la zone, mais leur absence de cohérence quant aux actions à mener après la catastrophe ne firent que plonger un peu plus les habitants dans une vulnérabilité déjà élevée avant même l'événement3.

L'exemple du quartier Los Incas, en périphérie nord-est de Cuzco, nous intéresse tout particulièrement pour appréhender dans le détail ce qui, depuis maintenant plusieurs décennies, a façonné cette construction du risque dans la ville andine. Plusieurs questions se posent alors :

- Comment historiquement, socialement, « urbanistiquement», les conditions amenant à des glissements de terrain successifs dans la zone nord-est depuis les années 1980, ont-elles été réunies, et quelles sont les parts de responsabilité des uns et des autres - habitants, représentants de quartier, municipalité, État et services décentralisés?

- Après la catastrophe, quelles réponses en matière de mitigation ont été apportées par ces différents acteurs, et en fonction de quelles représentations du risque souvent opposées institutionnelle, «culturelle» — se positionnent-ils ?

Une double approche, à la fois anthropologique et urbanistique, permet d'apporter des éléments de réponse.

Tout d'abord, on s'attachera à montrer comment à un type de catastrophe qui frappa le centre urbain de Cuzco (tremblement de terre de 1950), est venue se greffer une autre menace : le glissement de terrain, cette fois le long des pentes abruptes colonisées en périphérie. La responsabilité des pouvoirs publics dans la construction historique du risque est ainsi retracée dans le détail durant cette deuxième moitié du $20^{\mathrm{e}}$ siècle, à travers une présentation des choix politiques faits en matière de gestion urbaine, face à la ville en expansion.

Dans un deuxième temps, nous nous intéresserons aux parcours résidentiels des classes pauvres, venues des campagnes environnantes ou plus lointaines (autres provinces), pour rejoindre la ville

Comme San Sebastián ou San Jerónimo, situés à l'est.

2 Le quartier Los Incas, apparu en 1963 en périphérie nord-est de Cuzco, est divisé en neuf comités.

3 Ces questions de gouvernance et de gestion du risque traitées dans le cas d'étude du quartier Los Incas, s'inscrivent dans le vaste champ de recherche développé par I'Institut Universitaire d'Études en Développement et l'IP8 (Individual Project 8) à Genève. L'IUED/IP 8 sont inscrits dans le cadre du programme de coopération de la recherche suisse NCCR North-South. Le Pérou, la Bolivie et I'Argentine sont les trois pays latino-américains $d^{\prime}$ 'intervention de cette équipe pluridisciplinaire, dont les responsables Isabelle Milbert et Marc Hufty méritent ici d'être chaleureusement remerciés. Cet article, résultat de deux mois d'enquête dans le quartier Los Incas au début 2004, fait suite à l'étude Gobernancia y riesgos ambientales urbanos en Cusco réalisée par le Centro Bartolomé de Las Casas (2003), situé à Cuzco et coopérant avec l'IUED/IP8. 
de Cuzco, du centre aux périphéries. Nous pourrons constater alors que la recherche de plus d'espace habitable et d'une réduction des coûts du logement en se regroupant dans la famille étendue, l'emporte sur le «choix» d'une exposition plus faible au risque. Dans un contexte de crise interne après la catastrophe, quand les relais au sein du voisinage entre habitants et leurs représentants de quartier ne fonctionnent plus, l'action collective peut être abandonnée au profit d'un repli sur la parcelle familiale.

Enfin, nous étudierons la représentation du risque spécifique que se font les habitants indiens (quechua) et métis des périphéries urbaines, en fonction de leur rapport notamment à la terre considérée comme divinité agissante. Dans cette optique, nous préférerons le terme représentation à celui de perception. Comme le soulignent très justement Pirotte et al. (2000), la notion de perception implique qu'il y ait un risque concret, seul capable d'être pris en compte, opposé à un autre type de risque, plus imaginaire... Or, peut-on parler d'objectivité du risque ? Le terme de représentation permet d'assumer la complexité de l'objet, en s'appuyant sur les systèmes cognitifs spécifiques à chaque groupe étudié, en fonction notamment de leurs cultures et croyances propres.

Pour espérer améliorer leur sort face aux glissements de terrain à répétition, les habitants des périphéries en pente de Cuzco ont ainsi élaboré une « culture » du risque, particulière au contexte socio-politique de cette région péruvienne. À partir de cette étude de la construction du risque à Los Incas, dans la périphérie nord-est de Cuzco, au-delà d'une approche fonctionnaliste et culturaliste du risque, il s'agit donc bien de montrer comment les habitants se sont organisés face à une absence de volontarisme politique ou à des choix contradictoires chez les autorités débouchant sur l'immobilisme. L'approche anthropologique et urbanistique est dès lors mise au service d'une compréhension plus large des enjeux politiques entourant la gestion du risque, dans la ville andine.

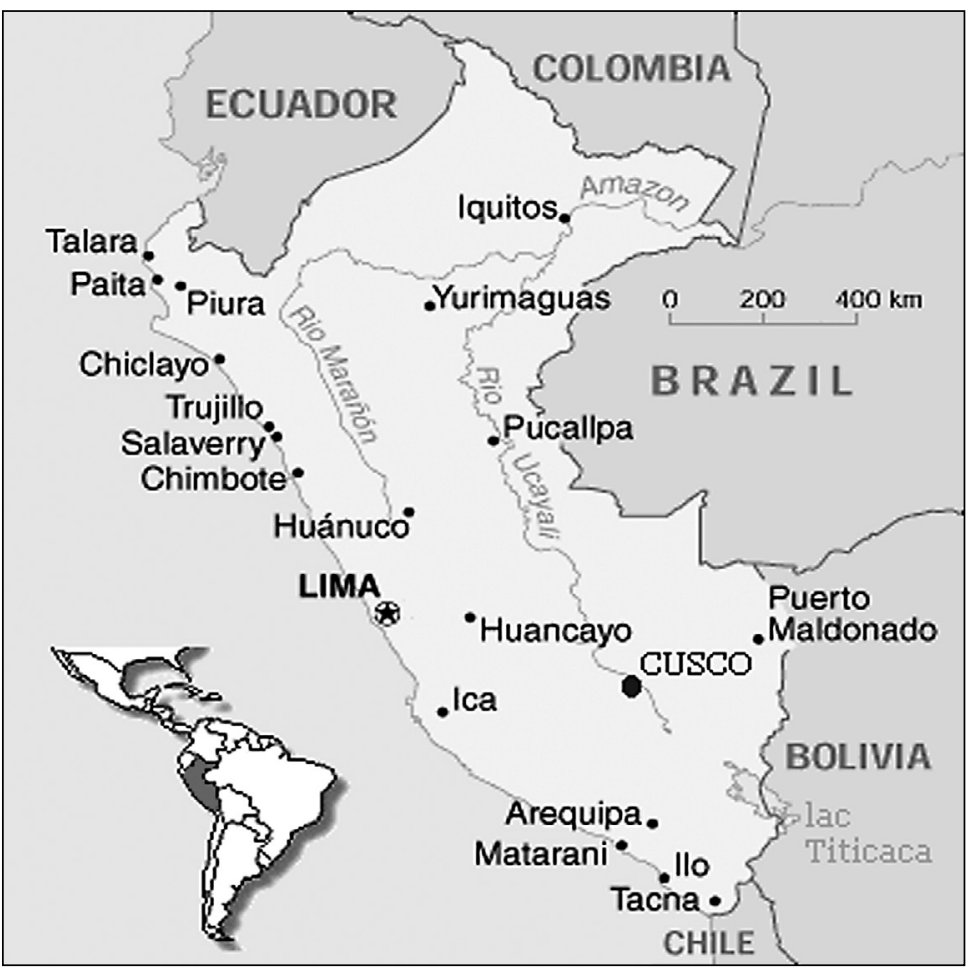

Figure 1 - Le Pérou et Cuzco, capitale de l'empire inca

(élaboration : Rey según www. americas-fr.com/geographie/ cartes/carte_perou.html - 24k) 


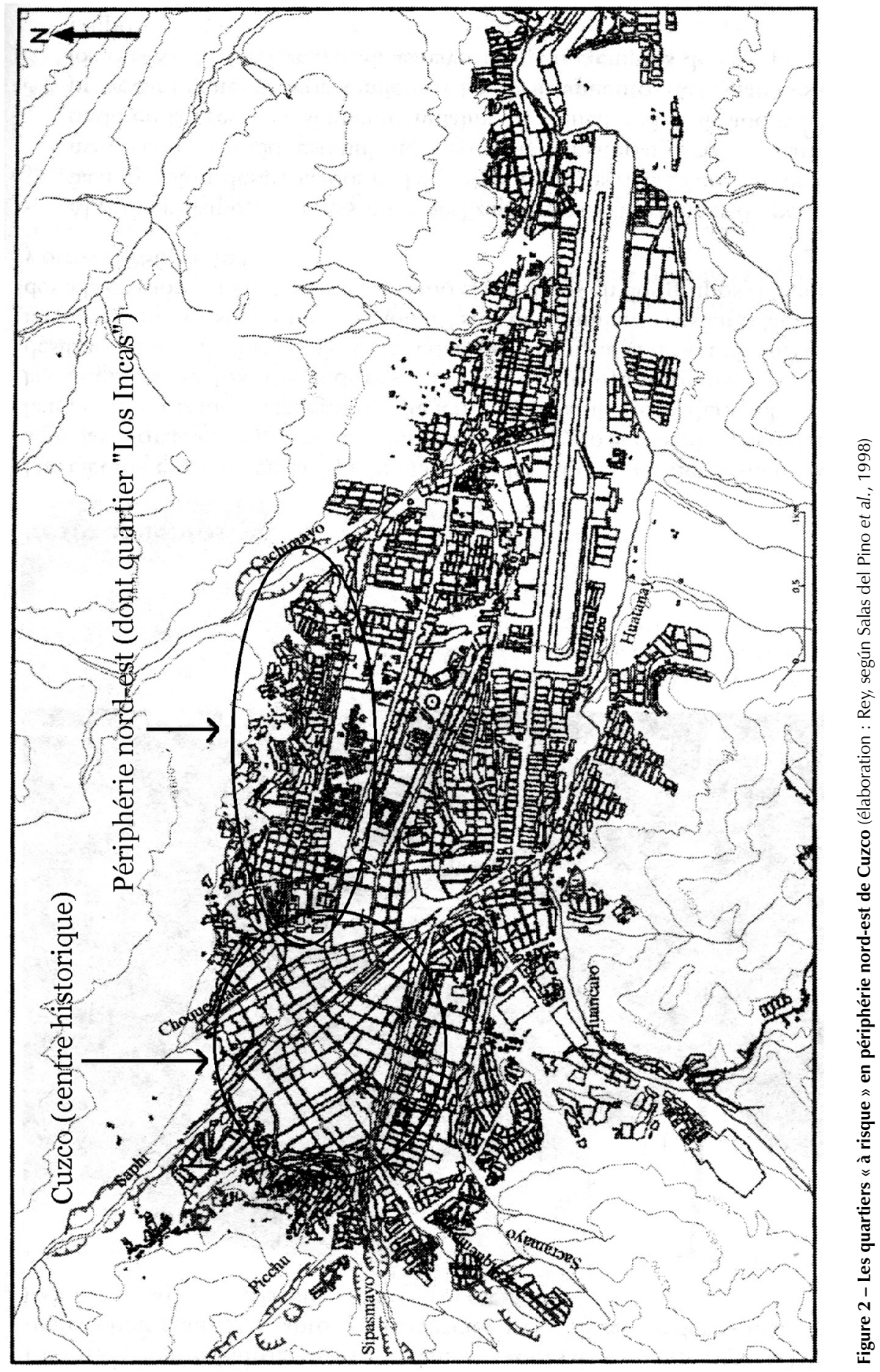


Nicolas Rey

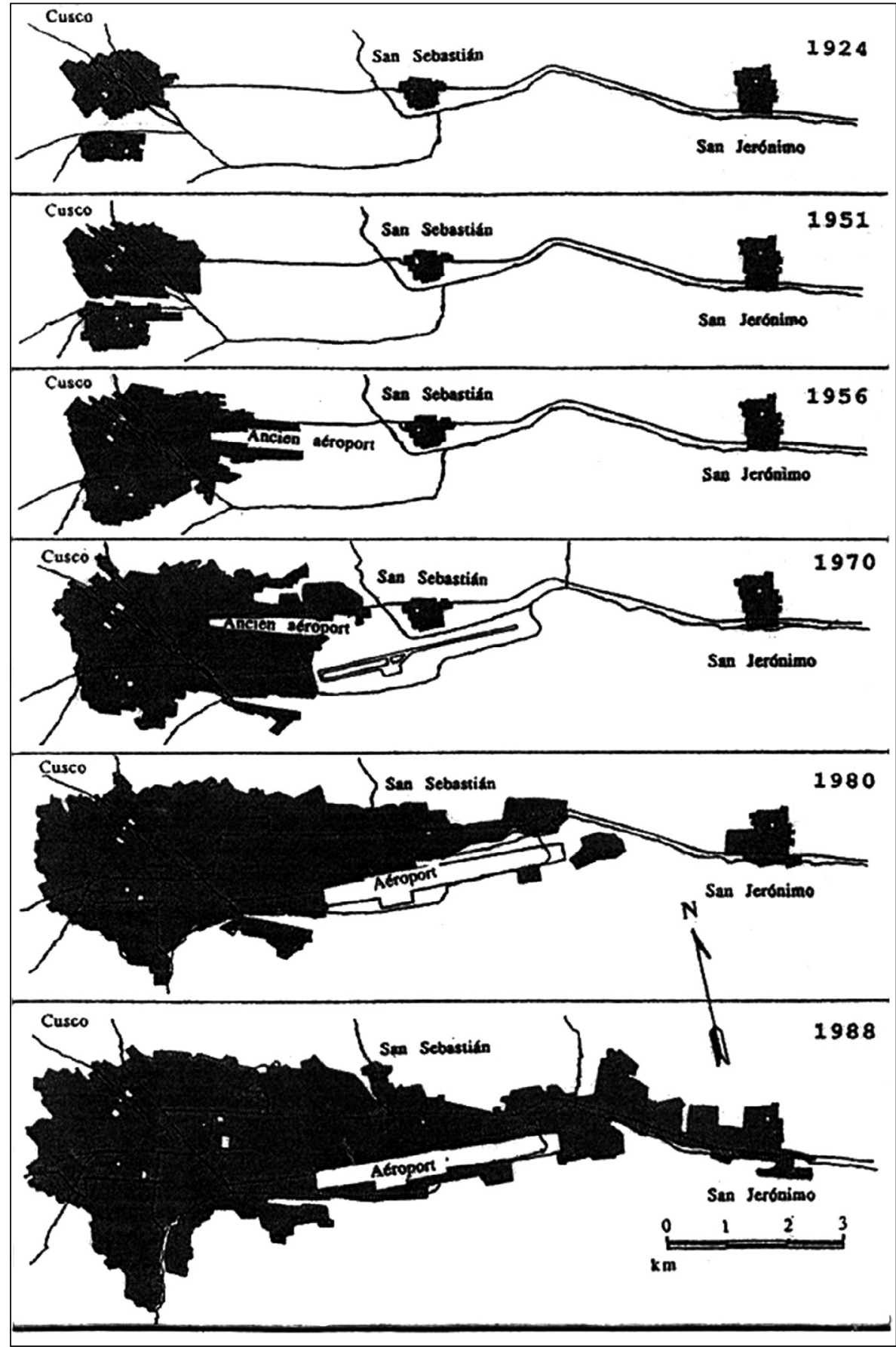

Figure 3 - L'expansion longitudinale de la ville selon le phénomène de «conurbation »

(d'après : Carreño, 1994) 


\section{LA CONSTRUCTION DU RISQUE PAR UNE EXPANSION URBAINE NON MAÎTRISÉE}

Avant le tremblement de terre de 1950, on comptait 216 hab./ha à Cuzco, ce qui représentait le taux de densité le plus élevé au niveau national (Marco Cortez, 1989). Les demandes adressées par les autorités locales à l'État afin de répondre à la surconcentration en ville par une politique volontariste de construction sociale (viviendas sociales), restaient lettre morte.

Il fallut attendre le tremblement de terre de 1950 pour voir finalement la ville s'étendre au-delà de son tracé colonial. Le bilan fut très lourd, transformant Cuzco en ville de barracas :

- sur un peu plus de 4000 maisons, seul un quart ne fut pas détruit ;

- environ 35000 personnes se retrouvèrent sans domicile. Vingt mille décidèrent de quitter Cuzco, tandis que le reste des sinistrés s'installa à différents points de la ville avec leurs tentes ou leurs maisons de fortune (barracas), sur les places, les avenues, les rues... (Marco Cortes, 1989).

La catastrophe eut au moins l'effet de pousser l'État à répondre enfin à la demande en logement à Cuzco. Une loi d'imposition sur le tabac va financer la reconstruction de la ville, et la participation internationale sera également conséquente. Le centre sera rénové, et la ville plus moderne va prendre vraiment forme, avec une expansion urbaine orientée délibérément vers l'est. La ville sera divisée en districts ${ }^{4}$, selon le Projet Pilote de 1952. La Réforme Agraire favorisa involontairement la vente de nombreux terrains, tant et si bien que l'offre devint même supérieure à la demande, certains propriétaires préférant se débarrasser de leurs vastes superficies à bon prix pour échapper à cette orientation politique étatique les défavorisant :

« En 1962/1963, la Réforme Agraire a favorisé la vente de terrains dans la vallée, près du Cuzco. Les propriétaires préféraient vendre leurs terres un bon prix à une compagnie immobilière ou le lotir eux-mêmes plutôt que de risquer de se le voir enlever par l'administration de la Réforme Agraire contre une indemnité qui serait moins importante » (Durand, $1970: 17$ ).

Les classes moyennes furent d'abord intéressées par l'expropriation, menée sous l'égide de l'État; puis les classes plus pauvres purent se reloger de façon moins planifiée, principalement à l'initiative privée des ordres religieux, de l'Assistance Publique 5 et des grands propriétaires terriens (hacendados), le long des pentes abruptes (laderas) :

«Après le tremblement de 1950, les problèmes urbains structurels s'amplifièrent. Près de $90 \%$ des logements en ville furent affectés par le séisme, ce qui provoqua le départ d'un grand nombre d'habitants vers d'autres aires, aujourd'hui périphériques. La plus grande déstructuration des espaces régionaux et la relation conflictuelle ville-campagne toujours non résolue, qui voit Cuzco ne pas être articulée de façon indépendante à son espace régional, surtout avec une absence de développement de l'industrie, furent une des causes qui motivèrent et accélérèrent la crise urbaine.

La prolongation de l'avenida de la Cultura dans les années 1950 sera un premier pas important dans l'implantation de quartiers vers le sud-est, qui s'accompagne d'un phénomène connu sous le nom de conurbation de l'espace urbain (...), sur les aires planes qui entourent la localité de San Sebastián. La majorité de ces terrains furent expropriés par l'État, qui devint le promoteur en logement pour les classes moyennes de la ville. La seconde pointe d'accroissement, dans les années 60, est caractérisée par l'apparition de nouveaux acteurs sociaux non officiels, ceux-là même qui donnèrent

4 Les districts sont aujourd'hui au nombre de cinq : San Sebastián, Huanchac, Cuzco, Santiago, et San Jerónimo.

5 La Beneficiencia Pública fut fondée en 1790, et rétablie après les indépendances, en 1833. Elle hérita des biens donnés aux hôpitaux de Cuzco à l'époque coloniale (Brisseau, 1981). 
lieu à l'extension de la ville clandestine, illégale et non planifiée (...) 》 (Quedena et al., 1994 : 83-84).

L'obligation de trouver un terrain où se loger va amener la création de nombreuses associations pour le logement (Asociacion Pro-Vivienda). La direction de chaque APV acheta de vastes superficies, cette fois de façon collective, les terrains à lotir étant redistribués ensuite entre les différents membres de l'association. De telles associations furent d'abord créées avec le souci d'obtenir les services comme l'eau ou l'électricité dans un second temps en faisant pression sur les pouvoirs publics. On va donc passer d'une occupation de terrains spontanée, à un type d'organisation plus structurée, mais en générant toujours les mêmes problèmes, les services et l'urbanisation échappant encore à toute planification :

«Les Associations sont régies par le Décret de Loi 13500 promulgué en 1961. Elles sont caractérisées par le fait que les associés acquièrent des terrains rustiques de la part de l'État, des Églises ou des propriétaires particuliers. Mais comme les coopératives, elles ne reçoivent pas I'appui de l'État, et le processus d'urbanisation est de leur responsabilité directe (...) 》 (UNSAAC, $1986: 28$ ).

Malgré une plus grande coopération entre propriétaires (État, Églises, particuliers) et habitants de ces zones qui achètent les terrains à travers la Asociacion Pro-Vivienda6, c'est donc toujours la politique de l'« après-coup » de la part des autorités qui prévaut, avec son lot de dysfonctionnements - discontinuité de la trame foncière, incohérence dans le système d'alimentation/évacuation des eaux, etc. — affectant à terme l'ensemble de la ville :

«L'occupation précède l'assainissement, aux normes, des terrains. Ce sera la nécessité de se doter des services de base et communaux qui, dans un second temps, amènera les occupants de lots à se regrouper en Associations ou Unions de Propriétaires ou lotisseurs. (...) Il apparaîtra également des problèmes liés à l'approbation de projets d'urbanisation : alignement des voies, faute de superficies de réserve pour les voies publiques, absence des $10 \%$ pour les espaces verts, des $2 \%$ pour l'État, etc. Les projets seront finalement approuvés après la régularisation ou la restructuration » (Marco Cortez, 1989 : 9).

Après le tremblement de terre de 1950 et la colonisation des laderas, les glissements de terrain vont affecter régulièrement la zone nord-est à partir des années 1980. Un type de risque en amène t-il un autre ? Certes, mais les grands responsables restent les pouvoirs publics qui, par faute de planification en direction des classes populaires, ont laissé les lotisseurs s'emparer des pentes, construisant ainsi des zones à haut risque en dehors de tout contrôle. Dans le quartier Los Incas, situé en périphérie nord-est de Cuzco, le long de pentes inclinées à plus de $30 \%$, les explications techniques le plus systématiquement avancées pour évoquer le risque urbain qui le menace, sont les suivantes :

- les maisons à majorité en adobe offrent peu de résistance aux secousses sismiques toujours systématiques dans la région (même si elles sont généralement de faible amplitude) ;

- la nature géologique des sols le long de ces pentes amplifie les ondes sismiques ;

- le système de drainage, tant en sous-sol qu'en surface, a été conçu en dehors de tout respect des normes de construction établies par les pouvoirs publics.

Mais c'est plus l'expansion de la ville sans contrôle, que le seul caractère « dangereux » en soi du site initial, qui a construit le risque à Cuzco :

« (...) ce n'est pas tant le site initial qui pose problème, mais l'expansion et la segmentation du périmètre urbanisé qui s'accompagne des modifications des sites dangereux dans l'espace et dans le temps au fur et à mesure du développement spatial de la ville, comme

6 D'autres dénominations vont s'imposer dans les années 1970 et 1980 pour qualifier ces quartiers dominant la ville : les quartiers apparus sous la présidence de Velasco seront dénommés pueblos jovenes. Ils seront désignés ensuite sous le terme de asentamientos humanos (établissements humains) dans les années 1980.

7 Le quartier est d'ailleurs traversé par une rue appelée à juste titre en quechua Anden Kawarina (« mirador des Andes »). 
I'illustre l'expansion de Cuzco au Pérou dans un couloir intra-montagneux devenu trop étroit » (Veyret, 2003 : 66).

C'est ainsi l'ensemble des habitants de la ville qui subit les conséquences d'une expansion urbaine non maîtrisée depuis cinquante ans : la principale desserte longitudinale de la ville, l'avenida de la Cultura, " coincée » dans une cuve montagneuse avec des voies secondaires (transversales notamment) sans continuité, est régulièrement inondée, ce qui rend les secours en cas de catastrophe extrêmement difficiles. Les quartiers de la zone moderne, pour les mêmes raisons de problème d'évacuation des eaux, sont régulièrement affectés par les crues (Carreño, $1994: 32$ ).

\section{LE RAPPROCHEMENT FAMILIAL EN PÉRIPHÉRIE, AU «PRIX » D'UN RISQUE PLUS GRAND}

Dans les années 1960, les ordres religieux, franciscains et dominicains, vont s'avérer être les principaux promoteurs fonciers dans l'expansion urbaine de Cuzco à l'est, le long des pentes. La Asociacion Pro-Vivienda Los Incas va ainsi apparaître en 1963, sur des terrains qui appartenaient aux Dominicains. Fortunato est un des premiers occupants, et le plus ancien encore présent dans le quartier. II nous raconte son installation à Los Incas :

«Los Incas a été fondé en 1963. Celui qui lotissait s'appelait Edgard Mostajo Sánchez, il était avocat. II vendait par lots, faisait des annonces à la radio. Aujourd'hui nous sommes 650 familles à Los Incas. Nous avons nivelé par ici, c'était de la terre friable. J'ai été le quatrième à m'installer ici. En 1962 j'ai rencontré ma femme, elle était de mon village, Acumayo. Là-bas j'allais démarcher à Maldonado, en chargeant du verre pour les travailleurs de l'or dans les mines... Pendant douze ans, en passant par le fleuve, les montagnes ; deux jours de chemin, pour m'y rendre. En 1963, on s'est mariés, et on est venus vivre ici. On a d'abord vécu à Huayrurupata, et les gens nous ont informé qu'il y avait un quartier avec des terrains à acheter, parce que louer ça coûte. Il y en a de deux cents, trois cents mètres carrés. Avec un ami, on a acheté ce lot, la moitié chacun, mais il y a eu une dispute. Il voulait tout accaparer et me virer. Nous sommes allés voir Mostajo, et il nous a divisé le terrain. Par la suite, je lui ai demandé de me connecter à son système d'évacuation des eaux, mais il n'a pas voulu. J'ai dû faire ma propre connexion. Ensuite, après que mes parents soient morts, ma sæur de Acumayo est venue dans l'année. Elle m'a dit: 'Tu as déjà une maison, trouves-moi un terrain '. Je suis allé demander pour elle. Mon frère aussi, il a quitté Lima pour venir vivre ici, avec sa famille. Il est d'abord venu vivre dans ma maison dans les années quatre-vingt, et après trois ans, je lui ai dit : ' tu ne peux pas rester là tout le temps. Tu dois te chercher un terrain '. De là, il est parti vivre à Garci Laso » (Fortunato, comité 6/ APV Los Incas, 31/03/04).

Garci Laso est un quartier contigu au comité 6 (Los Incas). D'un quartier à l'autre, les membres d'une même famille se sont étendus, à l'image de Fortunato, de son frère et de sa «soeur » (il s'agit en fait d'une cousine à lui), créant ainsi des réseaux sociaux favorisant la communication : une porte, en effet, reliait les parcelles des deux frères entre Los Incas et Garci Laso. Lorsque les glissements de terrain de 2001 et 2002 se sont produits dans le comité 6 , ces «passages » furent particulièrement mis à profit entre voisins et membres d'une même famille, situés sur des parcelles contiguës. Mais par crainte de vols, ou de viol de leur intimité, les propriétaires de ces passages préférèrent ensuite les fermer. Ainsi, la catastrophe peut dans un premier temps resserrer les liens qui s'étaient construits sur plusieurs années, pour ensuite les défaire. Les réseaux d'entraide mis en place à l'échelle de la parcelle entre les pionniers peuvent également, après rupture, amener aussi à cette construction du risque, par la multiplication de connections en alimentation/évacuation des eaux que cela implique, comme l'évoque Fortunato suite à la division qu'il opéra avec son ami de la première heure à Los Incas. Ainsi, la catastrophe est préparée bien avant qu'elle n'ait lieu, par la rupture de réseaux sociaux qui multiplie les 
incohérences sur le plan technique (connections au système d'alimentation/évacuation en eau). Dans le même temps, la catastrophe peut rompre des réseaux d'entraide qui fonctionnaient avant le désastre, depuis l'installation dans le quartier.

Comme les «pionniers » qui sont arrivés les premiers dans le quartier il y a quarante ans tel Fortunato, aujourd'hui encore on quitte les localités plus rurales pour rejoindre les autres membres de la famille, déjà installés en ville. Ce départ «s'organise » également comme autrefois : après s'être marié, on part à deux s'installer en ville pour mieux l'affronter, pour y chercher le mieux vivre et y fonder une famille. Les nouveaux venus sont alors dépendants du parent propriétaire de la parcelle. Le témoignage ci-dessous de Jesusa est particulièrement instructif lorsqu'elle évoque le contrôle social exercé sur elle par la famille de son mari chez qui elle est venue s'installer. Cet informateur se livre à une comparaison avec son ancien quartier de résidence, Rosas Pata, situé dans la ville plus formelle. Elle considère qu'elle avait plus de liberté « en ville » que dans le quartier périphérique de Los Incas où la famille de son mari et les voisins surveillent ses moindres faits et gestes. On a là un contrôle social qui va aller croissant de la part de sa belle-famille, en fonction de ses installations successives à Cuzco dans différents quartiers :

« Je viens de Ollantaytambo, où mes parents et mes frères faisaient de l'agriculture. J'ai vécu avec mon amoureux dès quatorze ans, et à seize je suis allée vivre à Huancaro, avec mon époux. C'était un mauvais quartier. Il y avait de nombreux voleurs qui rentraient dans les maisons, qui t'attendaient au tournant, te volaient tes vêtements. C'était comme ça. Quand il pleuvait, il y avait de la boue partout. L'eau sale du fleuve rentrait dans les maisons, les humidifiait, et après quelques années, elles tombaient.

Il y a huit ans, nous nous sommes installés à Rosas Pata. C'était mieux parce qu'il y avait le marché à côté. La propriétaire était la tante de mon époux et elle nous faisait payer moins cher. C'était pratiquement dans le centre de Cuzco, plus sûr, il n'y avait pas de voleurs. La maison était plus grande qu'à Huancaro, il n'y avait plus de boue dans les rues, c'était près des voitures. A Huancaro on avait une pièce, alors qu'à Rosas Pata on avait une pièce et une autre plus petite pour la cuisine... c'était plus pratique pour l'enfant, qui avait cinq ans, et plus prêt de l'école. Et mon époux était plus près de sa famille, à Rosas Pata.

Puis on est passés à Los Incas, il y a cinq ans. Car ici on ne paie pas de location, c'est la maison de ses parents. Ici nous avons trois pièces : une pour nous, une autre pour le garçon de treize ans et la fille de neuf ans, et la cuisine. Dans toute la maison [la parcelle], il y a trois familles en plus : mes belles-soeurs avec leurs époux et leurs enfants. En venant m'installer ici, je l'ai très mal vécu, parce que ma belle-soeur est très méchante avec moi, elle m'insulte : ' chèvre, chienne '... Elle ne voulait pas que je sois avec son frère. Elle voulait qu'il vive avec une amie à elle... Parfois quand il y a de l'eau, elle ne me laisse pas en prendre. Comme mon mari n'est jamais là, il ne sait pas tout ça. Également pour l'électricité, parce que eux ils l'utilisent plus avec tous leurs appareils : ils ont un réfrigérateur, alors je ne veux pas payer la moitié de l'électricité avec ce qu'ils utilisent... Je vivais plus tranquille à Rosas Pata, je sortais à n'importe quel moment, mais ici, tout le monde t'observe, à quelle heure tu sors, ce que tu manges...» (Jesusa, comité 6/ APV Los Incas, 31/03/04).

Le parcours résidentiel de Jesusa est très instructif, car il fait apparaître comment chaque installation auprès de la famille se fait en fonction de critères bien précis :

- chercher un plus grand confort, plus d'espace (on gagne une pièce à chaque déménagement) au fur et à mesure que s'agrandit la famille ;

- ne plus avoir à payer de location.

Mais ce que l'on gagne en espace habitable et en économies, se paie au prix :

- d'un contrôle social plus fort exercé par la famille étendue, qui peut se traduire aussi par un accès aux services sur la parcelle (eau, électricité) accaparé par les propriétaires de la parcelle, au détriment des nouveaux venus ; 
- d'un risque urbain et d'une vulnérabilité subies au quotidien (violence urbaine, maisons menaçant de s'écrouler, etc.).

Qu'est-ce qui pousse pour autant les habitants d'autres localités à rejoindre Cuzco ? La ville de Cuzco reste au centre d'un réseau urbain au niveau départemental très structuré. Au niveau de la province de Cuzco, l'accès aux services de base (eau, électricité) mais aussi l'alphabétisation notamment des femmes, sont deux à trois fois supérieurs à la moyenne nationale, et devancent de loin les autres provinces ${ }^{8}$. On quitte donc facilement les autres provinces du Pérou, mais aussi I'intérieur de la province de Cuzco, pour rejoindre cette ville centre, plus attractive :

«Cuzco (...) s'est affirmée comme le centre politico-administratif et économique, atteignant $24,2 \%$ de la population totale départementale et $54,6 \%$ de la population urbaine départementale en 1993 (...). Cuzco : c'est le centre et l'intermédiaire de presque toutes les dynamiques départementales. Sa hiérarchie politico-administrative, ses fonctions commerciales et financières, ainsi que sa longue histoire d'articulation aux niveaux régional et extrarégional, ont favorisé la concentration de services et l'ont converti en un grand pôle attractif à tous les niveaux, au-delà de ses seules fonctions marchandes. Cela a été renforcé par le flux aérien soutenu par le potentiel touristique régional, et en relation avec Lima, avec le marché et le centre politico-administratif national » (Hurtado \& Puerta, 1995 : 11).

Mais une fois installés dans les périphéries, les nouveaux arrivants attirés par les lumières de la ville, peuvent déchanter. En effet, les conditions d'accès aux services pour les plus pauvres, que ce soit en périphérie de Cuzco ou dans le centre historique, ne sont pas meilleures que les chiffres avancés à l'échelle nationale. D'après Riofrio (1996), les conditions de pauvreté et le manque de services (eau), touchent autant les pauvres installés dans les constructions coloniales et modernes du centre historique, que ceux ayant rejoint les zones à risque des périphéries, où les voies carrossables manquent cruellement. On rejoint donc la famille pour avoir de plus faibles coûts en logement et parfois plus d'espace, mais au prix d'un accès aux services limité et d'une exposition au risque élevée. Comme le souligne D’Ercole (1991) pour la ville de Popayán, en Colombie — qui connut un séisme à la suite duquel les pentes furent également comme pour Cuzco colonisées par les exclus du logement social ou du secteur capitaliste - le risque social est bien plus obsédant pour ces habitants, que le risque naturel. Et cet auteur d'ajouter (1991 : 452) que la vulnérabilité « n'est pas seulement liée aux conditions économiques et sociales, mais aussi à la manière dont les gens perçoivent le risque sismique ». Après enquête auprès d'habitants de Popayán ${ }^{9}$, le sentiment de sécurité qui se dégage est «trompeur » et constitue un facteur de risque en cas de nouvelle catastrophe ; en revanche cet optimisme n'est pas partagé par tous, notamment par les habitants les plus modestes. Si ces derniers dans leur quartier ne se sentent pas menacés, ils craignent dans le même temps les conséquences d'une nouvelle catastrophe à Popayan. Mais les habitants des zones qualifiées « à risque » ont aussi une représentation du risque qui est d'ordre stratégique ; ils savent habilement en faire un élément de pression sur les autorités dans leur confrontation pour l'occupation des rares zones disponibles : les secteurs en pente.

\section{3. «CULTURE » DU RISQUE CONTRE IMMOBILISME DES AUTORITÉS}

Dans la partie basse du comité 6/APV Los Incas, la plus touchée par les glissements de terrains successifs d'avril 2001 et janvier 2002, les enfants préfèrent emprunter les chemins de traverse

8 Selon les recensements nationaux de population et de logement de 1993 (INE), Cuzco s'élevait au premier rang pour l'accès à l'eau, à l'électricité, et pour l'alphabétisation des femmes, avec des taux avoisinant les $90 \%$, contre des moyennes nationales respectivement d'environ $45 \%, 25 \%$ et $65 \%$.

9 Réalisée par D'Ercole, avec l'appui du BRGM (Bureau de recherches géologiques et minières), d'Ingeominas et du Sena de Popayán. Presque tous les habitants enquêtés considèrent que la ville est mieux préparée à affronter un nouveau séisme, pour des raisons essentiellement techniques (maisons reconstruites, réparées et consolidées). 


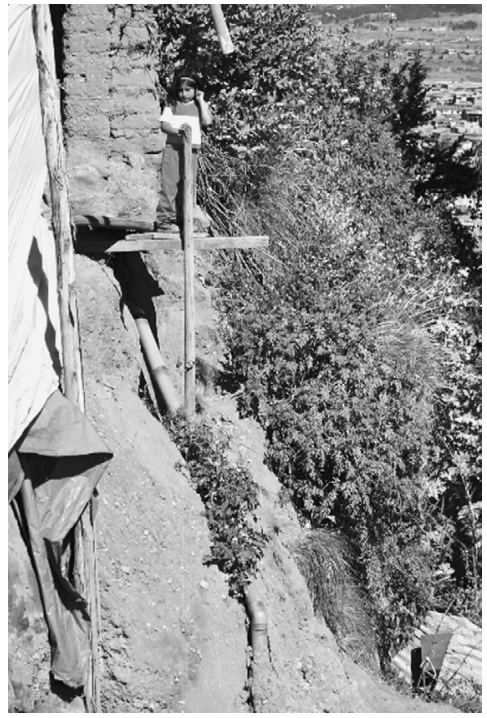

Figure 4 - Zone sinistrée du quartier Los Incas. Une fillette " joue» avec le risque, à l'entrée de ce qu'il reste de la maison familiale suite aux glissements de terrain de 2001 et 2002 (photo : Rey) entre les pans de murs effondrés et les tuyauteries éventrées, plutôt que de suivre les circuits tracés autour du vide laissé par la partie écroulée. Ils « jouent» à se faire peur, en repoussant les limites du danger. Mais est-ce uniquement, un jeu d'enfant ?

Durant ces événements, les habitants sont restés jusqu'au dernier moment dans leurs maisons: lorsque les murs ont commencé à s'effriter et à se lézarder, et que les vitres se sont fendillées, ils ont enfin décidé d'évacuer leur logement. Par la suite, ils sont revenus s'installer dans leur maison éventrée, en se plaçant en retrait des pièces situées en équilibre instable au-dessus du vide. Pour eux, il est hors de question de quitter le seul bien qu'ils possèdent, leur maison, fut-ce jusqu'au dernier moment... Le fait de vivre dans un lieu constamment menacé, et souvent affecté par la catastrophe, peut aussi amener une accoutumance, qui se traduit par une réponse de moins en moins efficace pour faire face au désastre, avant, ou pendant qu'il se produit :

"L'évaluation postcatastrophique est sans doute un des moyens les plus efficaces pour comprendre le passage du risque à la catastrophe, et donc proposer des mesures de prévention. (...) ce sont les études postcatastrophe qui ont révélé les lois concernant l'accoutumance au danger ; ces rapports montrent aussi la perte de mémoire classiquement observée des usagers et des décideurs. Voici ce qu'écrivait en 1890 un officier de l'instruction publique, Henry Vaschalde, à propos des crues et inondations en Languedoc : 'Plusieurs années se passent sans qu'il y ait de crues ; la confiance revient ; les maux passés sont oubliés, et chacun s'empare du domaine des eaux jusqu'à ce qu'une inondation comme celle du 22 septembre vienne détruire tous les travaux exécutés ' (Dauphiné, $2001: 252-253)$

Mais peut-on mettre en cause uniquement l'inconscience, ou la perte de mémoire ? Dans des conditions extrêmes d'existence, les habitants vivent au jour le jour, sans avoir toujours le luxe de pouvoir se projeter dans l'avenir. Dans les villes des pays en développement, que ce soit le long des pentes abruptes, ou près des cours d'eau comme à Lima (cf. exemple cidessous), le désastre rend les pauvres encore plus pauvres au quotidien (Maskrey, 1989) : les habitants ressortent encore plus vulnérables d'une première catastrophe, cette vulnérabilité en augmentation préparant les conditions d'un deuxième désastre, pouvant alors être plus ample. Catastrophe après catastrophe, les habitants sont donc de plus en plus vulnérables et exposés à de nouveaux désastres :

«Les inondations (...) affectent principalement les familles à bas revenus, qui ont les capacités de réponse les plus faibles et dont les conditions de vie sont celles d'un état d'urgence permanent, caractérisé par le manque d'eau potable, un habitat précaire et des revenus bas et instables. Dans ces cas là, les effets des (...) inondations ne sont qu'un aspect d'un désastre quotidien et permanent. Les catastrophes dans la vallée du Rímac représentent une dégradation continue de la vie de la population. La destruction de leurs maisons, la perte de leurs biens et l'interruption de leurs activités économiques augmente leur vulnérabilité. Sans la possibilité de retrouver de nouvelles zones sécurisées pour vivre, les gens reviennent occuper les secteurs dangereux » (Medina, 1994 : 273).

Si le désastre est social, la pauvreté en est le facteur le plus agissant. Et plus on est pauvre, plus la catastrophe annoncée sera amplifiée. En 2003, dans le cas du quartier Chocco, en périphérie sud-est de Cuzco, certains habitants qui avaient disposé leurs maisons au milieu du lit naturel d'écoulement du fleuve, ne purent réagir rapidement pour s'échapper lors de la soudaine montée des eaux. Le coût humain n'est jamais considéré par ces occupants de l'extrême, car nous sommes dans une dynamique de survie au jour le jour, empêchant de se projeter bien loin 
dans l'avenir (un toit et manger chaque jour sont les seules prérogatives qui font force de loi). II faut aussi bien prendre en compte le fait que les derniers migrants, arrivés récemment dans ces zones, sont aussi les moins avertis face aux catastrophes, et ce, pour deux raisons :

- tout d'abord, ils s'installent là où il y a encore de la place, donc le plus souvent, dans les zones les plus ingrates, et les plus dangereuses, qu'on leur réserve (la communauté préfère en effet se garder les terrains mieux situés, pas forcément les plus chers) ;

- la seconde raison est qu'ils n'ont pas encore pu mesurer la temporalité et l'intensité des catastrophes potentielles. Cette information peut d'ailleurs ne pas être divulguée par les responsables communautaires, au moment de la vente. Ainsi, à Chocco, un migrant arrivé en 2003 acheta son terrain près du fleuve traversant le quartier, sans se douter de la gravité du danger qui le menaçait. Dans l'année de son achat, sa maison et ses biens furent emportés par les crues.

Aujourd'hui, à Los Incas, trois maisons sont en équilibre instable au-dessus du vide. Parmi ces occupants, deux familles ont acheté leur maison il n'y a pas plus d'une dizaine d'années: les derniers arrivés, pas forcément les plus pauvres, sont en tout cas les plus mal lotis face au risque. Le temps de l'expérience et les réseaux d'information sont donc des données fondamentales à considérer dans la construction du risque. Comment les habitants de ces secteurs les plus touchés par les glissements de terrain en 2001 et 2002, et menaçant de s'effondrer à tout moment, ont-ils vécu la catastrophe, et pourquoi reviennent-ils s'installer là ? Julio, un de ces habitants en sursis, nous relate les événements du 24 janvier 2002, qui ont affecté son foyer :

« Je ne peux pas partir, car mes enfants vont au collège à côté, donc pour le transport, je préfère rester ici. La moitié de ma maison est fendue. La fissure n'a pas progressé après l'effondrement. La porte ne se ferme pas bien. Mais ici, le problème c'est l'eau. Si on me reloge avec l'eau là-bas, moi je partirai. Les autres à côté, ils ne veulent pas partir. Mais moi je ne peux plus vivre là, la maison glisse, il peut y avoir un tremblement de terre. En fait, il y avait un tuyau sous la terre qui s'est cassé, donc on voyait juste la sortie d'eau en surface mais on ne voyait rien en dessous. Si on avait réglé ça... ça fait cinq ans que par en dessous ça travaillait, et pire lorsque la pluie est arrivée : ça s'est mélangé avec cette poche d'eau d'en dessous. On s'est rendu compte de ça après le glissement de terrain.

Pour l'éboulement de 2002, c'était dix heures du soir, le vingt-quatre janvier. Mais un jour avant j'avais déjà remarqué que le chemin était fissuré, et la nuit, tout un bloc est parti. C'était l'anniversaire de ma fille ce jour là, pour ses vingt ans. Elle était venue fêter son anniversaire avec son mari. Finalement, elle est restée trois jours, car la situation était désespérante. On était dans l'expectative, si quelque chose se passait, prêts à courir. L'éboulement, ça a fait comme un volcan, les pierres, le bloc avec les escaliers... Les gens situés plus bas, à Los Portales, sont sortis en hurlant lorsque toute cette masse a défoncé leur mur. Les plus petits chez moi dormaient, les plus grands étaient en train d'observer. On avait prévu de sortir par derrière par les escaliers, pour aller chez le voisin : par là on a sorti nos affaires, le lendemain [de la catastrophe]. Le lendemain, la police nous a dit à tous qu'il fallait libérer les lieux et se transférer ailleurs. Un de mes fils est revenu s'installer dans la maison pour la surveiller, il ne voulait pas rester dans la maison louée plus haut. 'Ah si elle tombe, qu'elle tombe ', il disait... » (Julio, comité 6/ APV Los Incas, 09/03/04).

L'analyse du témoignage de Julio révèle plusieurs choses. Le statut de résidence principale, la présence sur place, et l'ancienneté de la propriété, sont mis en avant pour bénéficier d'une intervention extérieure afin d'être relogé. À cela vient se superposer le fait que les voisins immédiats de Julio déclarent ouvertement qu'ils ne veulent pas être relogés ailleurs... Julio cherche donc ostensiblement à se démarquer d'eux, en remettant en cause les revendications de ses voisins qui, ayant racheté à sa famille leur terrain et leur maison, ne sont pas en mesure légitimement selon lui d'imposer leur point de vue, au nom de tous. Il y a donc une différenciation sociospatiale très forte, au sein même du secteur touché par la catastrophe, qui détermine l'attitude de chacun, face au risque. 
Les rares interventions des habitants pour réduire le risque, peuvent même prêter à sourire : on dispose des planches sans les fixer, contre le mur du voisin situé plus haut, prêt à tomber... II n'y a donc pas de réelle prévention du risque, mais plutôt une gestion dans l'urgence : la mitigation (réduction du risque) durable serait-elle absente des consciences ? II semble que les habitants soient tout à fait conscients du risque qu'ils encourent. Leur « inaction » ne traduit pas une inconscience avérée ; au contraire, elle est une stratégie visant à faire réagir les autorités. Le manque d'attention que portèrent les hommes envers la Pacha Mama, la Terre Mère, lorsqu'ils urbanisèrent les quartiers périphériques, est également une explication avancée par certains habitants du quartier Los Incas, concernant les glissements de terrain qui se sont succédés. Le terme pachakuti, traduit aussi par « retour de la terre » à travers ces glissements de terrain, est alors évoqué, comme une réaction de la Pacha Mama... En Bolivie, ces termes ne sont plus connus de la population indienne. Ils seraient cependant réinvestis par les leaders communautaires et/ou des anthropologues développant un discours indigéniste, notamment en milieu urbain :

«Dans la société aymara, le juicio marque la fin d'un cycle et le début d'un autre. Ce bouleversement qui verra l'apparition d'une nouvelle humanité est interprété comme une inversion entre le monde du haut et le monde du bas (Harris, 1989), idée exprimée dans les documents anciens par le terme pachakuti, aujourd'hui inconnu des paysans rencontrés mais repris depuis peu dans les discours indianistes urbains » (Rivière, 1996 : 93).

Cette culture du risque semble avoir un objectif sous-jacent, et elle représente l'ultime issue pour les habitants, afin de faire pression sur les autorités : tout est fait comme s'il fallait en rajouter un peu plus à une situation déjà extrême, pour attirer l'attention de façon directe et sans détours, sur les conditions de vie « invivables» de ces habitants. Dire qu'ils en jouent n'est pas un vain mot. Lorsque les mères balancent leurs enfants de cinq ans à bout de bras au-dessus du vide, en les faisant pleurer, peut-être est-ce pour leur apprendre à dompter la peur de leur condition, pour mieux y faire face, ou encore pour m'interpeller, moi l'étranger, représentant à leurs yeux des ONG et autres organismes internationaux, capables d'intervenir sur ces secteurs ?... Les premiers mots qui me furent adressés par certains de ces habitants furent d'ailleurs : "vous voulez parler avec nous pour quoi faire, pour nous aider?»

De plus, les enfants montent les échelles reposant sans y être fixées sur des planchers flottant au-dessus du vide, sans armatures les reliant à la pente ; ils sont envoyés également plus bas par les parents qui les ignorent alors ostensiblement du regard, pour ramasser les ustensiles - seaux, vaisselle, linge - tombés dans la pente, dans des endroits inaccessibles... un faux pas, et c'est la chute dans le précipice formé par l'effondrement de l'escalier qui se trouvait là, avant la catastrophe.

Enfin, aucun escalier de rechange, même de fortune, n'a été reconstruit par ces habitants, qui chaque matin, chaque midi, chaque soir, font le va-et-vient entre chez eux et l'extérieur, au péril de leur vie...

Qu'attendent-ils d'ailleurs, au juste ? Avril 2001, avril 2004... après maintenant trois ans qu'a eu lieu le premier drame, et autant de temps de promesses non tenues par les autorités, que peuvent encore espérer les habitants comme amélioration de leur condition au quotidien ? "Après les pluies, nous commencerons les travaux », leur répètent les autorités, comme un leitmotiv. Les élections passent, et aux promesses électorales succède l'inaction, après le résultat des urnes. Les autorités intervenant sur la zone sont toutes d'accord pour l'évacuer, mais chacune apporte une réponse différente au problème posé.

La municipalité de Cuzco et le Ministère du Travail sont associés à travers le projet a trabajar urbano qui consiste dans la zone nord-est de la ville à consolider la zone, avec la construction à Los Incas d'un mur de contention qui est systématiquement reportée. Les dissensions politiques entre le gouvernement et la mairie sont d'ailleurs souvent avancées par ces deux acteurs pour justifier l'absence de prise de décision10. Chacun se renvoie donc la balle, et pendant ce temps,

10 Le premier des deux acteurs (le Ministère du Travail), «tolediste», du parti du président de la république de l'époque, accusant le second (la municipalité de Cuzco) — et vice-versa — d'être affilié à l'autre bord politique, celui de Fujimori (ancien chef de l'État péruvien). 
les lignes budgétaires tant au niveau de la municipalité, que du gouvernement, ont été utilisées à d'autres fins. En 2004, le Ministère du Travail a pris prétexte des fortes pluies qui ont affecté le sud du Pérou pour justifier à nouveau du report des travaux sur Los Incas.

Pour la Défense Civile décentralisée (région de Cuzco), la zone doit être évacuée. Ses ingénieurs déclarent cependant qu'une fois la zone consolidée par le projet a trabajar urbano, certains habitants seront alors convaincus que la zone aura été débarrassée de tout risque possible.

On constate donc que la politique de décentralisation menée par l'État rencontre des contradictions très fortes au niveau local, entre les décisions prises par ses services au niveau local (Defensa Civil de Cuzco), la municipalité, et les ministères. La décentralisation fut même, sous Fujimori, mise au service " du chef», déstructurant l'action collective et rendant les structures de quartier encore un peu plus dépendantes :

«Dès son arrivée à la présidence, A. Fujimori institue une extrême personnalisation du pouvoir. Les fréquentes visites qu'il effectue dans les barriadas en témoignent. Cette personnalisation du pouvoir, qui se manifeste par l'instauration de relations directes et clientélistes avec les pobladores, s'observe dans plusieurs domaines. En premier lieu, I'organisation administrative que le chef de l'État institue, et dont l'exemple le plus significatif est le ministère de la Présidence. Ensuite, la politique de démantèlement des structures d'action collective avec l'exemple du programme du verre de lait. (...) Au début des années 1990, l'obtention d'un service collectif, par exemple au bureau de la voirie, exige des démarches précises : soixante familles au minimum, et appartenant au même district, doivent se réunir afin de présenter leur revendication et de solliciter un crédit. Cette demande s'adresse au ministère de la Présidence, qui finance directement le projet. Ainsi, le poblador peut associer directement la construction d'une infrastructure à l'image du président » (Burgos, 2000 : 108-109).

\section{CONCLUSION}

Comme développé dans cet article, l'absence de volontarisme politique dans le domaine de la planification urbaine depuis le tremblement de terre de 1950 à Cuzco, ou le caractère contradictoire de certaines décisions prises par les autorités suite aux glissements de terrains se succédant depuis les années 1980 en périphérie nord-est de la ville, sont générateurs pour les habitants d'augmentation et du risque, et de la vulnérabilité. Dans le même temps, devant cette défection des pouvoirs publics, les habitants se sont rassemblés en associations pour organiser leur logement, avec de faibles moyens. Ils sont tombés dans le marché de la ville illégale, en s'installant dans les seules zones disponibles, à savoir les plus dangereuses (le long des pentes abruptes ou des courants d'eau en crue dès la période des pluies), sans respect des normes de construction en matière d'alignement de voirie, de système de drainage, ou de résistance des matériaux dans une région à forte activité sismique. Une véritable «culture » du risque va alors se développer, mêlant à la fois au premier abord résignation face à un destin qui s'acharne, voire attitude de trompe-la-mort, comme pour provoquer un peu plus la providence, ou encore retour à des croyances se réclamant du passé incaïque. En effet, face à l'action des hommes « modernes » pris dans le modèle urbain occidental dominant, la Terre (la Pacha mama) et les ancêtres incas morts pour préserver ce territoire contre l'expansion coloniale espagnole, se vengeraient, en réagissant de façon violente à travers les catastrophes frappant la ville (crues, glissements de terrain). Peut-on se contenter cependant d'une explication de type fonctionnaliste ou culturaliste pour appréhender dans sa complexité la construction du risque dans cette ville andine?

De plus, l'après catastrophe sans union pour faire face aux problèmes quotidiens qui affectent tous les habitants (le quartier n'en finit pas de s'affaisser après les glissements de terrain survenus), et l'abandon de l'action collective pour se replier sur la famille, sont propices à une augmentation sensible de la vulnérabilité de chacun. Cette vulnérabilité s'est accentuée suite au détournement par certains représentants de quartier de fonds alloués par les habitants pour les travaux d'ordre 
général. Le « pourrissement » de la situation est ainsi renforcé, plongeant un peu plus le secteur effondré, vers une nouvelle catastrophe. Les habitants seraient-ils à ce point inconscients en laissant leur quartier tomber en ruine?

Sur le terrain du quartier Los Incas, lorsque l'espoir s'est encore un peu plus éloigné en avril 2004 de voir s'améliorer la situation par une intervention des pouvoirs publics sur la partie affectée (glissements de terrain de 2001 et 2002), on peut observer qu'individuellement, certains habitants sont intervenus alors eux-mêmes pour prendre enfin en charge un minimum de travaux d'amélioration. Après que les habitants aient été informés du nouveau report du projet de consolidation de leur secteur effondré, certains parmi les plus menacés ont amorcé des travaux d'amélioration :

- les uns ont fermé avec des planches le terrassement qui marquait l'entrée de leur maison, où les enfants jouaient, au-dessus du vide ;

- les autres ont creusé dans la pente quelques marches, permettant ainsi de remonter dans les maisons en équilibre instable, en limitant les risques de faire une mauvaise chute.

L'inaction des habitants n'était donc qu'un leurre, destiné à faire pression sur les autorités...ou comment l'inaction est, de fait, action! Dire que les habitants de ces quartiers ne sont pas conscients du risque qu'ils encourent, $c^{\prime}$ est faire totalement fausse route, sans voir les stratégies cachées mais inhérentes, à leur attitude, jugée par d'aucuns, passive. Quant à la prévention, ce n'est pas à eux, mais aux autorités, de l'assumer. Il y a en réalité une conscience très aiguë du risque chez les habitants, et leur inaction de façade amenant un pourrissement de la situation (maisons qui tombent en ruine) est de fait, après analyse et recontextualisation, une action des plus volontaristes qu'il soit, pour précisément amener les autorités à prendre leurs responsabilités, à terme. Et si l'on peut sortir de la situation de confrontation entre pouvoirs publics et autorités, peut-être sera-t-il alors temps d'envisager le rapatriement des pratiques préventives populaires acquises au fil du temps par les habitants, vers les secteurs décisionnels :

« De tout temps, des systèmes d'entraide communautaire plus ou moins institutionnalisés, tel que le gotong royong dans les kampungs de Djakarta, ont permis d'éviter bien des victimes dans des incendies ou des inondations. Cette réalité sociale apparaît comme un élément très positif à partir duquel les autorités publiques sont tentées d'utiliser ces réseaux traditionnels pour ' apprivoiser le risque '. L'idée est d'informer et d'accoutumer les populations au risque et de créer de 'bons ' réflexes. (...) Il a souvent été remarqué que la pauvreté, la densité, les problèmes spécifiques liés à la gestion et l'absence d'accès à certains outils techniques accentuent la vulnérabilité des villes du sud, alors que peut-être, à l'inverse, ces dernières possèderaient une spécificité en matière de réponse sociale à la crise (...)» (Milbert, $2003: 325)$.

Pour I'heure, le risque demeure un enjeu politique entre habitants et autorités, construit avant, pendant et après la catastrophe. «Action » et « inaction » sont donc à recontextualiser de façon très précise, en fonction des enjeux et intérêts sous-jacents voyant s'opposer les différents acteurs intervenant sur la zone sinistrée. D'un côté, la démarche de l'anthropologue vise alors à comprendre comment le «traditionnel» est instrumentalisé par les habitants, pour amener les autorités à réduire le risque. La culture du risque en étant étudiée en fonction du système de croyance propre aux sociétés andines, ne doit donc pas tomber dans le piège de l'assessorat ethnique. De l'autre, il s'agit de percevoir comment le risque naturel peut être instrumentalisé par les autorités, afin d'entretenir une dépendance entre administrés et leurs représentants. Cette dépendance repose sur une politique de l'urgence : on n'intervient qu'après-coup, une fois la catastrophe passée. La vulnérabilité des habitants est telle qu'ils ne peuvent refuser tout type d'aide, aussi minime soit-elle, afin de panser les plaies du désastre. Cette relation clientéliste entre habitants et élus, construite durant les décennies d'installation le long des laderas, est finalement consolidée une fois la catastrophe réalisée : culture du risque chez les habitants contre immobilisme par l'action contradictoire des autorités, ne font que perpétuer un tel système. 
Si l'on souhaite appréhender au plus près ces enjeux qui opposent habitants et autorités, entre représentation du risque dans les secteurs menacés et intervention des pouvoirs publics dans l'espace urbain et péri-urbain, le recours à une approche de type socio-spatiale, offre des outils d'analyse permettant de mieux cerner la construction du risque dans sa complexité. L'approche sociologique, développée brillamment depuis les années 1980 par les chercheurs du réseau latino-américain La Red, emmenés notamment par Maskrey, a évolué récemment vers une méthodologie de type microsociologique ou anthropologique, avec la constitution d'une véritable anthropologie des catastrophes, notamment à travers les travaux d'Oliver-Smith (2002) ou encore de Stein (2002). Et les recherches menées par les géographes français dans les années 1990 (D'Ercole, Thouret, Chardon, etc.), ont eu le mérite d'articuler l'approche spatiale avec celle plus sociale, du risque. Reste à la recherche sur le système risque de mobiliser davantage les chercheurs européens, nord ou sud-américains, pour éviter qu'ils ne rejoignent le champ de leurs recherches de prédilection (Lavell, 1993), après les grandes catastrophes11.

\section{Références citées}

BRISSEAU, J., 1981 - Le Cuzco dans sa région : étude de l'aire d'influence d'une ville andine, 571 p. ; Lima : Intitut Français d'Études Andines. Série Travaux de l'IFEA, 16.

BURGOS, D., 2000 - Villa El Salvador : un bilan de la participation politique. Problèmes d'Amérique latine, $\mathbf{n}^{\circ} 38$ : 101-116.

CARREÑO, C., 1994 - Risques naturels et développement urbain dans la ville andine de Cuzco, Pérou. Revue de Géographie Alpine, $\mathbf{n}^{\circ}$ 4, Tome LXXXII : 27-45.

Centro Bartolomé de Las Casas, 2003 - Gobernancia y riesgos ambientales urbanos en Cusco ; Cusco.

CHARDON, A.-C. \& THOURET, J.-C., 1994 - Cartographie d'une vulnérabilité de la population citadine face aux risque naturels: le cas de Manizales. Mappemonde, 4 : 37-40 ; Montpellier.

D’ERCOLE, R., 1991 - Vulnérabilité des populations face au risque volcanique, le cas de la région du volcan Cotopaxi (Équateur). Thèse de doctorat ; Grenoble : Université Joseph Fourier.

DAUPHINÉ, A., 2001 - Risques et catastrophes. Observer, spatialiser, comprendre, gérer, 288 p. ; Paris : Armand Colin.

DURAND, G., 1970 - La banlieue est du Cuzco et son expansion contemporaine. Mémoire de maîtrise de Géographie ; Paris : Université de Paris 8.

HURTADO, I. \& PUERTA, M., 1995 - Las tendencias del crecimiento urbano en el departamento de Cusco. Crónicas Urbanas, 4: 5-16; Cusco : Crónicas urbanas.

LAVELL, A., 1993 - Ciencias sociales y desastres naturales en América latina: un encuentro inconcluso. In: Los desastres no son naturales (A. Maskrey, ed.) : 135-154; Bogotá: La red de estudios sociales en prevención de desastres en Amérique Latina, La RedTecnología intermedia. ITDG.

MARCO CORTEZ, A., 1989 - La historia que no fue contada. ¿Quiénes le dieron al Cusco la forma que ahora tiene ? Crónicas Urbanas, 1: 4-12; Cusco : Crónicas urbanas.

11 Cf. les tremblements de terre de Mexico, San Salvador, Guatemala, ou la catastrophe du Nevado de Ruiz, qui ont mobilisé la communauté scientifique internationale (Colombie). 
MASKREY, A., 1989 - El manejo popular de los desastres naturales : estudios de vulnerabilidad y mitigación, 208 p. ; Lima : ITDG.

MEDINA, J., 1994 - Experiencias de mitigación de desastres. In : Viviendo en riesgo : comunidades vulnerables y prevención de desastres en América Latina (A. Lavell, ed.) : 267-282 ; Bogotá: Facultad latinoamericana de ciencias sociales-FLACSO, Red de estudios sociales en prevención de desastres en América Latina, La Red-Centro de prevención de desastres naturales en Centro América. CEPREDENAC.

MILBERT, I., 2003 - Vulnérabilité et résilience des métropoles : «elles sont si fragiles ». In : Développement durable et aménagement du territoire (A. Da Cunha, ed.) : 313330 ; Lausanne : Presses Polytechniques et Universitaires Romandes.

OLIVER-SMITH, A., 2002 - Catastrophe and culture: the anthropology of disaster, 312 p. ; Santa Fe: School of American Research Press.

PIROTTE, C., HUSSON, B. \& GRÜNEWALD, F. (eds.), 2000 - Entre urgence et développement, pratiques humanitaires en question, 237 p. ; Paris : Karthala.

QUEDENA, E., ESTRADA, E., APAZA, D., PAREDES, P., ARTEAGA, Y. \& QUIÑONEZ, L., 1994 Los retos del desarrollo agro-urbano, el caso de San Jerónimo-Cusco, 208 p. ; Cusco : Centro Guaman Poma de Ayala.

RIOFRIO, G., 1996 - Lima: Mega city and mega-problem. In : The Mega-City in Latin America (Gilbert A., ed.) : 155-172 ; Tokyo : The United Nations University Press.

RIVIÈRE, G., 1997 - Bolivie : le pentecôtisme dans la société aymara des hauts-plateaux. Problèmes d'Amérique latine, 24 : 81-102 ; Paris.

SHERBONDY, J., 1986 - Los Ceques: un código de canales en el Cusco incaico. Allpanchis Phuturinqa, 27 : 39-74; Sicuani : IPA.

STEIN, H. F., 2002 - Toward an applied anthropology of disaster: learning from disasters experience, method and theory. Illness, crisis and loss, 10 (2) : 154-163.

UNSAAC, 1986 - Problemática urbana, vivienda y tecnología mejorada en el departamento del CusCo, 185 p. ; Cusco : UNSAAC.

VEYRET, Y., 2003 - Les risques, 256 p. ; Paris : SEDES. 